\title{
Ketogenic diet-mediated steroid metabolism reprogramming improves the immune microenvironment and myelin growth of spinal cord injury rats through gene analysis and co-expression network analysis
}

\section{Hong Zeng}

Peking University Third Hospital

Yao Lu

Peking University Third Hospital

Meng jie Huang

Peking University Third Hospital

Yan yan Yang

Peking University Third Hospital

Hua yi Xing

Peking University Third Hospital

Xiao xie Liu

Peking University Third Hospital

Mouwang Zhou ( $\nabla$ zhoumouwang@163.com )

Peking University Third Hospital https://orcid.org/0000-0003-1553-0613

\section{Research}

Keywords: Spinal cord injury, Ketogenic diet, RNA-seq, steroid Biosynthesis, metabolic reprogramming, immune microenvironment

Posted Date: October 20th, 2020

DOI: https://doi.org/10.21203/rs.3.rs-53258/v2

License: (c) (1) This work is licensed under a Creative Commons Attribution 4.0 International License. Read Full License 


\section{Abstract}

Background The ketogenic diet has been widely used in the treatment of various nervous system and metabolic-related diseases. Our previous research found that a ketogenic diet exerts a protective effect and promotes functional recovery after spinal cord injury. However, the mechanism of treatment is still unclear. In this study, different dietary feeding methods were used to detect myelin expression and gene level changes between different groups.

Result We established 15 RNA-Seq cDNA libraries and divided them into 4 groups (SCI_KD, SCI_SD, Sham_KD and Sham_SD), and a total of 32,883 genes were detected by RNA-seq. First, KEGG pathway enrichment of upregulated differentially expressed genes (DEGs) in the SCI_KD and SCI_SD groups and GSEA analysis of the two groups found that a ketogenic diet significantly improved the steroid anabolic pathway in rats with spinal cord injury. Through cluster analysis, PPI analysis and visualization of iPath metabolic pathways, Sqle, Sc5d, Cyp51, Dhcr24, Msmo1, Hsd17b7, and Fdft1 changed significantly in the pathway. Second, through WGCNA analysis, all samples are placed in a gene network to analyse the correlation between gene modules and phenotypes. After module analysis, GO function and KEGG analysis showed that rats fed a ketogenic diet showed significantly reduced immune-related pathways, including those associated with immune and infectious diseases.

Conclusion A ketogenic diet may improve the immune microenvironment and myelin growth in rats with spinal cord injury through reprogramming of steroid metabolism.

\section{Background}

Spinal cord injury ( $\mathrm{SCl})$ is a devastating event that can lead to neurological deficits and motor dysfunction [1-2]. The dysfunction of motor and sensory functions after $\mathrm{SCl}$ is caused by the following two processes: primary injury and secondary injury. Primary injury results from irreversible mechanical damage to the spinal cord, which directly damages various tissue components. In contrast, secondary injury is a cascade of effects triggered by primary injury that presents as a variety of biochemical and pathological events, such as oedema, inflammation and apoptosis [3-4]. Current treatment strategies for $\mathrm{SCl}$ aim to protect neurons from secondary damage [5-6]. However, therapy is only effective in a limited scope [7]. Thus, understanding the molecular changes in $\mathrm{SCl}$ after therapy could lead to improved treatment of patients with SCl.

A ketogenic diet $(K D)$ is a high-fat, low-carbohydrate, low-protein diet that results in prominent production of ketone bodies (KB: $\beta$-hydroxybutyrate [BHB], acetoacetate [ACA] and acetone) [8]. Furthermore, KD has been validated as an effective non-pharmacological preventive treatment for drug-resistant epilepsy [910]. Interestingly, recent studies have suggested that a KD might also be beneficial to humans with neurological disorders, such as Parkinson's disease, Alzheimer's disease, multiple sclerosis, and traumatic brain injury [11-13]. Multiple reports have revealed that a KD may exert neuroprotective effects after SCI 
[14-19]. For example, Tetzlaff et al. found that KD improves forelimb motor function after SCI [14]. Zhu et al. revealed that a KD exerted neuroprotective effects by attenuating oxidative stress in SCI rats [18-19]. Finally, our study recently reported that a ketogenic diet improved motor function by activating Nrf2 and suppressing NF-KB signalling pathways to attenuate oxidative stress and inflammation after spinal cord injury [17]. However, the underlying mechanisms by which a KD provides neuroprotection after $\mathrm{SCl}$ still not fully understood.

Based on the above questions, in this study, we performed RNA sequencing to examine the changes in gene expression of spinal cord tissue in Sprague-Dawley (SD) rats treated with different methods. We screened out differentially expressed genes and comprehensively detected genes with important biological significance through gene set enrichment analysis (GSEA) at the overall level [20]. Weighted gene co-expression network analysis (WGCNA) was used to establish a gene co-expression network to explore the relationship between the gene network and the KD diet phenotype [21]. These data promote further understanding of the new genes and related signal pathways and how KD treatment improves the recovery of motor function in $\mathrm{SCl}$ rats, providing new guidance for clinical treatment.

\section{Results}

\section{Experimental procedures and animal conditions}

The entire experimental process is shown in Fig. 1A. To established a C7 spinal cord hemi-contusion model, unilateral C7 laminectomies were performed using a Precision Systems \& Instrumentation-IH Impactor according to the manufacturer's instructions (Additional file 1: Fig. S1A). SD rats were anaesthetized with sodium pentobarbital to expose the spinal cord. We fixed the C6-T1 vertebrae rigidly in a frame tilted at a $25.0^{\circ}$ angle and then used the Infinite Horizon impactor to deliver a set contusion force of $150 \mathrm{k}$ dyne (Additional file 1: Fig. S1B); the contusion forces were monitored by computer (Additional file 1: Fig. S1C). An average of $150 \mathrm{k}$ dyne contusion force was obtained for each sample. These results indicated that the $\mathrm{C} 7$ spinal cord hemi-contusion model was successful. Using myelin staining, the myelin sheath area in the SCl_KD group was significantly larger than in the SCI_SD group (Fig. 1B-C), indicating that $\mathrm{SCl}$ rats protected or promoted myelin sheath growth at the injured site after feeding with a ketogenic diet.

\section{Differentially expressed mRNAs}

To further understand the effects and mechanism of the ketogenic diet on SCl, RNA-seq was performed on each group with 5 biological replicate samples, in which 5 samples were degraded and eliminated. To explore transcription factor expression in SD rats treated with KD under spinal cord injury, we established 15 RNA-Seq cDNA libraries divided into 4 groups (SCI_KD, SCI_SD, Sham_KD, and Sham_SD). A summary 
of the sequence assembly after Illumina sequencing is presented in Additional file 2: Table S1. The average raw reads was 23295214.7 , the Q30 basic mass fraction of the sample was $100 \%$, and the error rate $(\%)$ was 0 , meeting the requirements for subsequent analysis. Using a comparison of the transcriptome and reference genome, greater than $82 \%$ of reads were uniquely mapped; these result meet the subsequent analysis requirements (Additional file 3: Table S2). In short, the sequencing data is suitable for subsequent data analysis.

A total of 32,883 genes were detected by RNA-seq, and Venn analysis between samples showed the number of expressed genes in each group (Fig. 2A). We first used an unsupervised classification methodprincipal component analysis (PCA) to characterize the differences between the gene expression profiles of the 15 samples. Fig. $2 \mathrm{C}$ shows that the biologically replicated samples can be divided into the following 4 groups: Sham_SD1-3, Sham_KD1-4,SCI_SD1-3 and SCI_KD1-5. The PCA model shows that the maximum variation in $\mathrm{PC} 1$ can explain $16.92 \%$ of the variation, while principal component 2 explains $9.85 \%$ of the variation [11]. In Fig. 2B, the scatter plot of differential genes between the SCI_KD and SCl_SD groups shows that 463 genes are differentially expressed between the two groups, among which 184 differentially expressed genes are upregulated and 279 differentially expressed genes are downregulated. The top 50 upregulated and downregulated genes are listed in Additional file 4: Table S3 and Additional file 5: Table S4, respectively.

\section{Reprogramming Steroid Biosynthesis and Metabolism in SCI Rats on KD}

Compared with the SCl_SD group, the KD diet group upregulated DEGs in many enrichment pathways, such as the steroid synthesis pathway in lipid metabolism (path ID: map00100, q-value $=5.82043 \mathrm{E}-08$ ), biosynthesis of unsaturated fatty acid, and terpenoid backbone biosynthesisand, etc. A bubble chart of the differential genes in the top 20 most enriched pathways is provided (Fig. 3A). The downregulated DEGs from the KD diet group were significantly enriched in ribosomes, lysosomes, osteoclast differentiation, proteasome, tuberculosis, and antigen processing and display. Fig. 3B shows the KEGG enrichment distribution of the differentially expressed genes.

To further understand the biological functions of the SCI_SD and SCI_KD DEGs, we performed gene set enrichment analysis (GSEA) of the sample sets based on the C2 MSigDB database, which includes the Broad Institute recombined gene sets. The selected gene set was tested, and it was found that SCI_KD was significantly negatively correlated with inflammatory cytokines and their receptors (NES values are -1.44 and -1.46 , respectively; Additional file 6: Fig. S2), suggesting that a ketogenic diet reduces the lethal function of spinal cord injury, which echoes the results of our previous studies. Similarly, based on the KEGG functional annotation, we found that SCI_KD was significantly positively correlated with the steroid 
synthesis pathway (NES is 1.62; Figure 3C) by GSEA analysis, which further shows that the ketogenic diet is involved in the metabolic reprogramming of steroids in $\mathrm{SCl}$ rats, which is an improvement by the KD. An important mechanism of SCl function may be related to reducing metabolic responses. iPath3.0 was used to visually analyse the enriched steroid metabolism-related genes, as shown in Additional file 7: Fig. S3, which shows that the steroid metabolism DEGs in the biological system transmit information.

\section{Cluster analysis and PPI analysis of DEGs in the steroid synthesis pathway}

Figure 3D shows that the Sqle (squalene epoxidase), Sc5d (sterol-C5-desaturase), Cyp51 (cytochrome P450, family 51), Dhcr24 (24-dehydrocholesterol reductase), Msmo1 (methylsterol monooxygenase 1), Hsd17b7 (hydroxysteroid (17- $\beta$ ) dehydrogenase 7), and Fdft 1 (farnesyl diphosphate farnesyltransferase 1) genes were obviously highly expressed in the SCI_KD group. DEGs correspond to interaction relationships for network integration and allow us to identify key pathways in the interaction network according to indicators such as the connectivity between genes (Fig. 3E).

\section{WGCNA and Hub module identification and analysis}

According to the KEGG enrichment analysis of gene differential expression and the gene set enrichment analysis, the mean values showed that $\mathrm{SCl}$ rats in the ketogenic diet group significantly promote steroid anabolism, which fully indicates that the ketogenic diet may be protective by mediating steroid metabolism reprogramming. Perhaps by changing some physiological functions or pathways, we can analyse the SCI_KD, SCI_SD, Sham_KD, and Sham_SD results in a gene network (WGCNA), and fully understand the roles of a ketogenic diet and normal diet on genetic changes in SD rats. Genes that are commonly expressed in the four groups are placed in the same gene network through the WGCNA algorithm. A total of 32,883 mRNAs were screened by WGCNA, and a total of 5,163 mRNAs were used for subsequent analysis after pre-processing and filtering, such as excluding deletions and outliers. Fig. 4A and $4 \mathrm{~B}$ show the scale-free fitness curve and the average connectivity curve, respectively. The scale-free fitness curve is in a smooth position and the power exponent is a weighted $\beta$ value; thus, the soft threshold value is set to 6 and the scale-free topological index is 0.9 . Therefore, the network conforms to the power law distribution and is closer to the actual biological network state. The resulting gene tree diagram and the colours in the corresponding modules are shown in (Additional file 8: Fig. S4A-B), with the number of mRNAs for each module shown in Attached file 9: Table S5.

After the data was pre-processed, the genes were classified, and genes with similar expression patterns are divided into categories. These genes categories are called modules. We performed module clustering and module correlation analysis for the obtained modules, as shown in Fig. 4D. Interaction relationships were analysed in the six different modules, and a network heat map of the correlation between the modules and the phenotype was drawn (Fig. 4C). The results show that the modules are independent of 
one another, which indicates the relative independence of gene expression in different modules. Here, we mainly analysed the correlation modules between the ketogenic diet- and standard diet-fed rats. Among them, the MEgreen module is highly related to KD and the MEblue module is highly related to SD. We then calculated the correlation coefficient between each gene in the module and specific phenotype data to obtain the gene significance value (GS); this was combined with the MM value of each gene in the module (i.e., kME, eigengene-based connectivity) to obtain the MM-GS scatter plot. The stronger the correlation, the closer the relationship between the module and the phenotype; moreover, correlations with a lower $\mathrm{p}$-value are more reliable. This analysis shows that MEgreen is an important module in the KD diet, while MEblue is an important module in the SD diet (Additional file 8: Fig. S4C-F).

\section{Functional annotation analysis of hub modular genes}

To explore the biological functions of the KD diet and SD diet modules (green and blue, respectively) and compare the differences in their biological functions, we performed GO and KEGG enrichment analysis with annotation and visualization. GO function annotation analysis classifies DEGs using different perspectives, such as the biological processes involved, the components that constitute cells, and the molecular functions achieved. Figure $5 \mathrm{~A}$ shows the $\mathrm{GO}$ annotation functions of the MEgreen module and Figure $5 \mathrm{~B}$ shows the $\mathrm{GO}$ annotation functions of the MEblue module. Different from other groups, there was a significant positive correlation between $\mathrm{SCl}$ rats with normal diet and MEblue module. GO function analysis immune system processes were expressed in biological processes, which indicated that immune system significantly affected $\mathrm{SCl}$ rats, and immune system processes included all process of inflammation-related pathways, congenital and acquired immune expression changes.

Similarly, KEGG annotation analysis was also performed on the green and blue modules. The results show that the KEGG annotation molecule in the MEblue module significantly expresses immune system, immune system and infectious diseases: viruses and other immune disease pathways among subtypes. This module mainly focuses on rats with normal diet of $\mathrm{SCl}$, which is consistent with the GO function analysis results. Compared with MEblue module, in terms of expression ratio, immune system, immune system and infectious diseases: the sub-categories of viruses and other immune disease pathways are significantly down-regulated, and the module is related to the ketogenic diet (Additional file 10. Fig. S5).

\section{Multi-gene set KEGG enrichment analysis and gene interaction network analysis}

The Immune system and Infectious diseases: Viral and Immune diseases pathways were used as pathways of interest for multi-gene KEGG enrichment analysis, and Fig. 6A shows the KEGG enrichment results of the MEgreen module and Fig. 6B shows the KEGG enrichment results of the MEblue module. The MEgreen module is primarily a module closely related to the ketogenic diet rats while the MEblue 
module is mainly a module closely linked to the normal diet rats, especially the normal diet injury rats. The results of the KEGG enrichment signal pathways and intersection of the top 20 pathways for the two modules show that only the Viral myocarditis and Phagosome pathways are present in both modules. The MEgreen module mainly activates innate immune-related pathways, such as the Toll-like receptor signalling pathway and the NOD-like receptor signalling pathway. These results indicate that MEgreen and MEblue act on different immune pathways. The WGCNA and functional analysis showed that rats fed with a ketogenic diet showed a reduction in immune system pathway processes, including immune diseases, infectious diseases and other immune microenvironments.

\section{Discussion}

The ketogenic diet has been widely used in the treatment of a variety of neurological and metabolicrelated diseases, especially epilepsy [22-23]. In recent years, some studies, including previous studies performed by our team, have shown that the ketogenic diet has a protective effect after spinal cord injury by increasing the spontaneous movement and fine manipulation of SCl animal models [17, 24-25]. However, the mechanism of treatment is still unclear. In this study, myelin expression and transcriptome level changes between different groups were detected in rats under different dietary feeding methods. This is the first study to use RNA-seq to understand and identify differentially expressed genes in SCl rats fed a ketogenic diet and then examine which biological signal pathways can explain the protective effects of a KD diet on $\mathrm{SCl}$ rats.

In this study, we used a variety of analytical methods to mine and interpret entire transcriptome RNA sequencing data, including differentially expressed genes and functional enrichment analysis between groups, gene set enrichment analysis between groups, iPath metabolic pathway analysis, gene cluster analysis, WGCNA and module analysis, module function analysis and gene interaction network analysis. From single gene expression analysis to changes in the overall gene network, a comprehensive analysis of the changes in gene expression patterns under a ketogenic diet can provide a deeper understanding of changes in gene transcription and signal pathways by a KD for the treatment of spinal cord injury.

From a local perspective, we used KEGG pathway enrichment of upregulated DEGs in the SCI_KD and SCI_SD groups and GSEA analysis of these groups to reach a consistent conclusion that KD significantly increased the steroid anabolic pathway in rats with spinal cord injury. Through cluster analysis, PPI analysis and the iPath metabolic pathway visualization, we concluded that Sqle, Sc5d, Cyp51, Dhcr24, Msmo1, Hsd17b7, and Fdft1 changed significantly in the pathway. Steroid biosynthesis appears to play a pivotal role in SCl. 
The ketogenic diet is a high-fat, low-carbohydrate and moderate protein diet. Its purpose is to reduce carbohydrate intake and replace it with lipids while ensuring a sufficient protein supply [26]. This programme was proposed by Wilder in 1921 for the treatment of epilepsy. It was later used for a variety of neurological diseases, such as Alzheimer's disease, Parkinson's disease, etc. [27-28]. Although it is known that a KD promotes high ketone body levels, high fat, low carbohydrates, low calories and other "immediacy" properties [29-30], this study suggests that the ketogenic diet may mediate steroid metabolism reprogramming to treat spinal cord injury rats This study is the first to reach this conclusion. Steroids include sterols (such as cholesterol, lanosterol, sitosterol, stigmasterol, and ergosterol), bile acids and bile alcohols, steroid hormones (such as adrenal cortex hormones, androgens, and oestrogen), insect ecdysone, cardiac glycosides, saponins, and toad poison [31-33]. Eating a high-fat/low-carbohydrate ketogenic diet can cause the liver to produce ketones. In the brain, ketone bodies (such as $\beta$ hydroxybutyrate) contribute to sterol synthesis [34], which is essential for the growth of myelin membranes [55 (35)]. Studies have shown that diabetic patients can significantly increase HDL cholesterol and lower blood pressure through a 90-day KD diet, while LDL cholesterol shows no significant difference [36]. However, long-term ketogenic diet increases the risk of type 2 diabetes and metabolic syndrome. This study explored the relevant mechanism of short-term $\mathrm{KD}$ treatment in $\mathrm{SCl}$ rats [37]. Additionally, studies have found that HDLc showed small increases after ketogenic phases, but there was no significant change over 12 months [38].

This study also found that myelin areas were significantly larger in $\mathrm{SCl}$ rats after being fed a KD than SCl rats in the normal diet group. A recent study by Stumpf found that a ketogenic diet can improve axon defects and promote myelination in Pelizaeus-Merzbacher disease. A high-fat/low-carbohydrate ketogenic diet can restore the integrity of oligodendrocytes and increase CNS myelin. Lipids (such as ketone bodies) easily enter the CNS without destroying the blood-brain barrier or blood-spinal cord barrier [39]. Ketone bodies produced by a normal diet are not able to enter the CNS to enhance the myelin membrane. These conclusions indicate that a ketogenic diet mediates steroid metabolism reprogramming after spinal cord injury, facilitates myelin membrane synthesis, promotes myelin sheath regeneration, plays a unique role in neuroprotection, and does not destroy the blood spinal cord barrier function. It is well known that the blood spinal cord barrier is an important barrier for the spinal cord to maintain neuroimmune balance.

This study combined 4 groups of rat genes into the same gene network through WGCNA analysis to analyse the correlation between gene modules and phenotype. Through module identification and analysis as well as GO function analysis and KEGG pathway analysis, we showed that rats fed a ketogenic diet displayed significantly reduced immune-related pathways, including immune diseases and infectious diseases. In addition, examining the SCI_KD and SCI_SD groups data and existing MSigDB analysed by GSEA, we concluded that SCI_KD is significantly negatively correlated with inflammatory 
cytokines and their receptors. These results indicate that KD treatment can improve the body's immunity level and reduce infection and inflammation.

In $\mathrm{SCl}$ secondary injury, inflammation is an important and main pathophysiological process. After $\mathrm{SCl}$, ischaemia-reperfusion injury, endothelial cell injury and homeostasis disorders jointly initiate the inflammatory cascade reaction, and spinal cord innate immune cells (microglia and astrocytes) [40] and infiltrating white blood cells (neutrophils and macrophages) are activated [40-41]. The activated inflammatory cells release a large number of inflammatory factors to further expand the damage and increase the time and space of the damage response. Our research studies have shown that KD reduces or inhibits neutrophils in the plasma of SCl rats and inhibits the classic NF-kB pro-inflammatory signalling pathway at the injury site, which corresponds to the results of this study [17]. Steroid synthesis

is also closely related to SCI neuroinflammation, and steroid synthesis hormones [42], oestrogen [43-44], saponin [45], tauroursodeoxycholic acid [46] and other steroid components in the spinal cord injury all have anti-inflammatory properties. Fig. 8A-B show the KEGG signalling pathway at the intersection of the immune microenvironment of a normal diet and a ketogenic diet, respectively. The normal diet mainly activates innate immune-related pathways, such as the Toll-like receptor signalling pathway and NOD-like receptor signalling pathway. This study cannot directly prove a correlation between ketogenic dietmediated reprogramming of steroid metabolism and neuroimmunity, which is the limitation of the study. The significantly expressed genes in the steroid synthesis pathway need to be further examined in future studies to determine if they are related to $\mathrm{SCl}$ efficacy.

\section{Conclusions}

This study is the first to demonstrate that the ketogenic diet significantly promotes the reprogramming of steroid metabolism in the treatment of $\mathrm{SCl}$ al the transcriptional level. These changes are beneficial for myelin sheath growth and promote nerve function. Moreover, a comprehensive analysis of the ketogenic diet through WGCNA improves the $\mathrm{SCl}$ immune microenvironment and reduces inflammation. Therefore, we believe that the ketogenic diet may improve the immune microenvironment and myelin growth in rats with spinal cord injury through reprogramming of steroid metabolism.

\section{Methods}

\section{Animals and diets}

All animal experiments conformed to the Guide for the Care and Use of Laboratory Animals (NIH Publications No. 8023, revised 1978). All procedures and euthanasia performed in this study were approved by the Ethics Committee for Animal Experiments of the Department of Laboratory Animal Science, Peking University Health Science Center (NO. LA201456, 24 February 2014). 
We randomly divided 32 8-week-old male Sprague-Dawley rats (300-360 g) into the following four groups ( $n=8$ in each group): the control group was undamaged and divided into the standard diet group

(Sham_SD) and the ketogenic diet group (Sham_KD), while the SCl rats received either SD (SCI_SD group) or KD (SCI_KD group), 20 rats were used for RNAseq and 12 rats were used for histopathological examination of fixed specimens. All rats were reared at $21^{\circ} \mathrm{C}$ with a light/dark cycle of $12 / 12$ hours, and food and water were provided freely. Rats in the SCI_KD group were fed a KD (70 g/kg body weight/day), and the ratio of fat to carbohydrate and protein was 4:1 (fat: $50.5 \mathrm{~g} / 100 \mathrm{~g} \mathrm{KD}$; carbohydrate: $23.6 \mathrm{~g} / 100$; four hours after SCl, protein content: $5.5 \mathrm{~g} / 100 \mathrm{~g} \mathrm{KD}$; dietary fibre: $16.1 \mathrm{~g} / 100 \mathrm{~g}$ KD (Shenzhen Zeneca Inc., Shenzhen, China)). The rats in the Sham_SD group and SCI_SD group were fed normal carbohydrates. All groups had free water supply.

\section{C7 spinal cord semi-contusion}

A C7 hemi-contusion was performed in the two $\mathrm{SCl}$ groups as described previously for a different site of contusion [20]. Briefly, rats were anaesthetized with sodium pentobarbital after a unilateral (left or right side, chosen randomly) C7 laminectomy, and the C6-T1 vertebrae were rigidly fixed in a frame tilted at a $25.0^{\circ}$ angle. We then used the Infinite Horizon impactor to deliver a set contusion force of 150 kdynes [21]. The $\mathrm{C7}$ hemi-contusion is hereafter described as 'SCl.' Rats were fed for four weeks according to the above grouping and diet plan, and spinal cord tissue was sampled after four weeks.

\section{EC myelin stain}

Spinal cord tissue was placed in a fully automatic tissue dehydrator for an overnight dehydration programme (xylene, alcohol isocratic dehydration), embedded in paraffin, and cut it into 5 - $\mu \mathrm{m}$ continuous sections with a microtome after cooling overnight at $40^{\circ} \mathrm{C}$. The tissues were soaked in xylene I and II for 30 minutes each, after which they were soaked in $100 \%, 100 \%, 95 \%, 95 \%$, and $80 \%$ alcohol for 5 minutes each and then rinsed twice with double-distilled water. The tissues were then stained with triochrome cyanine (EC) for 15-20 minutes before undergoing ferric chloride (5.6\%) differentiation for a specific time under a microscope. We made sure not to over-differentiate, as the staining would be too light. Afterwards, the samples were dehydrated and mounted with neutral gum.

\section{RNA isolation, RNA-Seq Library Construction and Sequencing}

We randomly blindly divide 20 SD rats into 4 groups, namely SCI_KD, SCI_SD, Sham_KD and Sham_SD. Total RNA was extracted from a 1.6-cm spinal cord segment at the centre of the impact site with the Trizol reagent (Invitrogen, Thermo Fisher Scientific Inc., USA) and subjected to RNA-seq analysis to assess gene expression ( $n=5)$. Genomic DNA was removed using DNase I. Agarose gels ( $1 \%$ ) were used 
to monitor RNA degradation and contamination. A NanoPhotometer® spectrophotometer (IMPLEN, CA, USA) was used to check RNA purity. The Qubit ${ }^{\circledR}$ RNA Assay Kit was used to measure the RNA concentration on a Qubit $\AA^{\circledR}$ 2.0 Fluorometer (Life Technologies, CA, USA). The RNA Nano 6000 Assay Kit was used to assess the RNA integrity on a Bioanalyser 2100 system (Agilent Technologies, CA, USA). RNA sequencing of 5 biological replicate samples was performed for each group, and 5 samples were degraded and eliminated. Therefore, 15 RNA-Seq cDNA libraries were established.

1) We utilized two methods to treat the total RNA. Oligo(dT) magnetic beads were used to select mRNAs with polyA tails. rRNAs were hybridized with DNA probes and then digested to make DNA/RNA hybrid strand, followed by DNase I reaction to remove DNA probes. The target RNAs were then obtained by purification. 2) The target RNA was fragmented and then reverse transcribed into double-stranded cDNA (dscDNA) with N6 random primers. 3) The dscDNA was end-repaired with phosphates at the $5^{\prime}$ end and sticky 'A's at the 3' end, after which the sequence was ligated to an adaptor with sticky 'T's at the 3 ' end of the dscDNA. 4) Two specific primers were used to amplify the ligation product. 5) The PCR products were denatured with heat and the single strand DNA was cyclized with a splint oligo and DNA ligase. 6) Finally, the prepared library was sequenced with the Illumina HiSeq xten $(2 \times 150$-bp read length, Illumina, San Diego, CA, USA). The sequencing programmes were performed by BGI Company (China, Shenzhen).

\section{Quality control and expression level}

The raw paired-end reads were trimmed and subjected to quality control by SeqPrep (https://github.com/jstjohn/SeqPrep) and Sickle (https://github.com/najoshi/sickle) with default parameters. The paired-end clean reads were aligned to the Rattus_norvegicus reference genome (Rnor_6.0) using the default parameters in TopHat (http://tophat.cbcb.umd.edu/, version2.1.1) [47]. The reference genome and gene model annotation files were directly downloaded from genome website (http://www.ensembl.org/Rattus_norvegicus/Info/Index). RSEM (http://deweylab.biostat.wisc.Edu/rsem/) [48] was used to quantify the gene abundances. To identify DEGs between two different samples, the expression levels of each transcript were calculated based on the number transcript reads per million readings (TPM, per million transcript reads). The reads were then normalized using DEseq2 v. 1.24 .0 (http://bioconductor.org/packages/stats/bioc/DESeq2/) [49] and the statistical significance of the differentially expressed genes was assessed. The following default parameters and criteria were used here: Benjamini \& Hochberg $(\mathrm{BH})$ p-adjust $<0.05$ and $|\log 2 \mathrm{FC}| \geq 1$ after multiple comparisons. In the initial data exploration, we performed principal component analysis (PCA) to directly calculate the coefficient of variation between the groups in $\mathrm{R}$ prcomp.

\section{Gene function annotation analysis}


The genes and transcripts are aligned to six databases (NR, Swiss-Prot, Pfam, EggNOG, GO, and KEGG) to obtain comprehensive annotation information. The DIAMOND software (https://github.com/bbuchfink/diamond) [50]was used to sequence the genes and transcripts with the NR (ftp://ftp.ncbi.nlm.nih.gov/blast/db/), Swiss-Prot (ftp://ftp.uniprot .org/pub/databases), and EggNOG (http://eggnogdb.embl.de/\#/app/home) databases [51]. Sequence alignment was performed with the BLAST2GO and GO database, followed by alignment with the Pfam database using the HMMER software (ftp://selab.janelia.org/pub/software/hmmer3/3.0/hmmer-3.0.tar.gz) [52]. GO (Gene Ontology, http://www.geneontology.org/) is a database established by the Gene Ontology Federation. Its purpose is to standardize the biological terminology of genes and gene products in different databases and to determine gene and protein functions. Using the GO database, the genes in the selected gene set can be classified according to the biological process (BP) they participate in, the cell component (CC), and the molecular function (MF) they achieve. The results of KEGG Orthology (Kyoto Encyclopaedia of Genes and Genomes, http://www.genome.jp/kegg/) were obtained using KOBAS2.1. KEGG is a knowledge base that systematically analyses gene functions and links genomic information and functional information. Using the KEGG database, the genes in the gene set can be classified according to the pathways involved or the functions they perform [53].

\section{KEGG pathway enrichment analysis}

The KEGG PATHWAY enrichment analysis was performed on the gene set using an R script in KOBAS (http://kobas.cbi.pku.edu.cn/home.do) [53]. The BH method was used to calculate the corrected $\mathrm{p}$ value. The threshold of the corrected $p$ value (Corrected P-Value) is 0.05 . The KEGG pathway that meets the conditions showing that the pathway was significantly enriched for the gene set.

\section{iPath metabolic pathway analysis}

iPath3.0 (http://pathways.embl.de) was used to visually analyse the metabolic pathways and facilitate observation of the metabolic pathway information of differentially expressed genes in the entire biological system. Nodes represent various biochemical molecules and lines represent biochemical reactions. The overall metabolism of the biological system includes 146 metabolism-related KEGG pathways, 22 regulatory-related KEGG pathways, and 58 KEGG pathways involved in the biosynthesis of secondary metabolites. The nodes in the figure represent different compounds and the boundaries represent different enzymatic reactions. iPath3.0 can outline the biosynthesis and important regulatory pathways of secondary metabolites and easily identify complex metabolic pathways [54].

\section{Gene Set Enrichment Analysis (GSEA)}


GSEA is performed on a predefined set of genes, usually from functional annotations or the results of previous experiments (included in MSigDB, version 6.2, http://software.broadinstitute.org/gsea/downloads.jsp), and organizes the genes according to two sample types. The GSEA sorts the degree of differential expression and then checks whether the gene set clusters at the top or bottom of the ranking list. the first round of enrichment is based on $\mathrm{C} 2$, which includes curated gene sets (from online pathway databases, publications in PubMed, and knowledge of domain experts). The second enrichment is based on the results of KEGG functional annotation in this study. For the analysis results, we generally think that gene sets with the following characteristics are meaningful: $|\mathrm{NES}|>1$, NOM p-val $<0.05$, and FDR q-val $<0.25$.

\section{Protein-protein interaction analysis (PPI)}

We used an online search tool (STRING database, version 10.5; http://string-db.org/) to search for interacting genes to construct a protein-protein interaction (PPI) network based on the extensive functions of the genome-wide data [55].

\section{Weighted Gene Co-expression Network Analysis (WGCNA)}

The co-expression network is constructed using the WGCNA (v1.47) package in R [21]. WGCNA is an undirected, weighted network. "Unweighted network" means that the correlation between genes can only be 0 or 1.0 means that the two genes are not related, while 1 means they are related. A "weighted network" and genes are not only related or not but also their correlation value is recorded. The value is the weight (correlation) of the connection between genes. The soft threshold ( $\beta$ power) in the co-expression network is the expression of each gene at a specific time or space and is regarded as a node. To obtain the correlation between genes, it is necessary to calculate the correlation coefficient (Person Coefficient) any two genes; this allows us to know whether the expression profiles of two genes are similar. In this study, the $\beta$ power $=6$.

1) The module refers to the grouping of genes with similar expression patterns. Genes of a specific type below to a module.

2) An eigengene is defined as the first principal component of a given module. It is the most representative expression pattern of the module. It is an indicator that approximately represents the expression status of all genes in a module but is not a real gene.

3) A hub module refers to a module that is highly related to phenotypic data or closely related to research.

4) Gene significance (GS), or a measure of gene importance, is the correlation between gene expression patterns and the characteristics of a sample. Generally, the larger the correlation value, the more important the gene, which can be evaluated as the log conversion of the p-value. The correlation index 
between a gene and a specific pathway can also be the correlation between a gene and a specific phenotypic feature.

5) Module membership ( $M M)$, also known as the eigengene-based connectivity (kME), is the correlation between the expression pattern of a gene and a characteristic gene in the module. The larger the value, the greater the possibility that the gene belongs to the module.

Genes with the highest MM and the highest GS are genes with high significance (hub genes). All these analyses were performed using commands implemented in the WGCNA software package with the following parameters: minModuleSize $=30$, minKMEtoStay $=0.3$, and mergeCutHeight $=0.2$.

\section{Modular gene expression correlation analysis}

After a gene of interest is obtained based on the correlation of gene expression, the correlation coefficient between genes is determined using the spearman correlation algorithm. The correlation coefficient threshold is 0.5 and the significance level is the $p$ value after $\mathrm{BH}$ correction (Corrected $p$-value) $\leq 0.05$, and these values were drawn into a visual network diagram. In the figure, the nodes represent genes and the connections between nodes represent the correlation between gene expression. The larger the node, the greater the expression correlation between the genes.

\section{Multi-gene set KEGG pathway enrichment analysis}

After performing KEGG analysis on the different gene sets, we utilized the intersection of the selected gene sets for the display and adjusted the significance level to the corrected $p$-value $\leq 0.5$ as the threshold to display the top 20 pathways.

\section{Statistical Analysis}

The data are expressed as the mean \pm standard deviation (mean \pm SD). The statistical analysis was performed with GraphPad Prism 7.0 (GraphPad Software Inc., San Diego, CA). The Student's t-test was used for comparisons between two groups. A p value $<0.05$ was considered statistically significant. * $p$ $<0.05, * * p<0.01$, *** $p<0.001$, and ${ }^{\star \star \star *} p<0.0001$.

\section{Abbreviations}

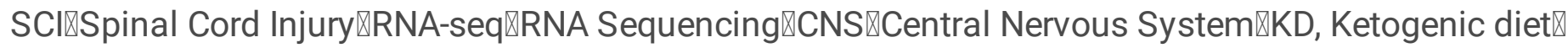

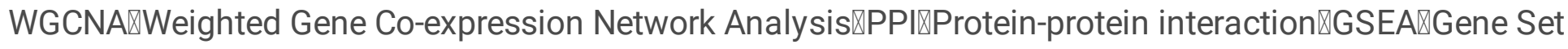




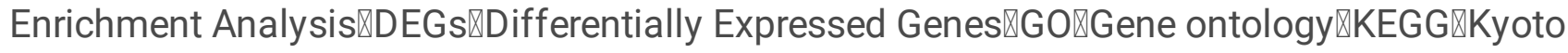

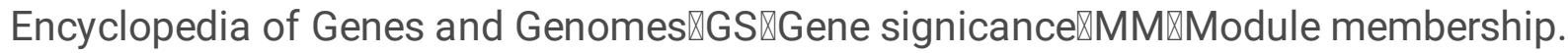

\section{Declarations}

\section{Acknowledgements}

We thank Dr. Wolfram Tetzlaff and his colleague Jie Liu (International Collaboration on Repair Discoveries [ICORD], Blusson Spinal Cord Center, Vancouver, British Columbia, Canada; Department of Zoology, University of British Columbia, Vancouver, British Columbia, Canada) for the hemi-contusion model. We are grateful for the technical support provided by Peking University Third Hospital Central Laboratory. We also thank to the Bioinformatics Technology Service provided by Biotech Information Platform of Major Biotechnology Co., Ltd of China.

\section{Funding}

This research was funded by National Natural Science Foundation of China (Projects of International Cooperation and Exchanges NSFC, Grant No. 81261120563).

\section{Availability of data and materials}

RNA-Seq raw data have been deposited in the NCBI Sequence Read Archive (SRA, https://submit.ncbi.nIm.nih.gov/subs/sra/SUB7836712/overview). Accession IDs for Rattus norvegicus BioProject $=$ PRJNA648739 $;$ BioSample $=$ SAMN15646629 - SAMN15646643 (15 objects) $₫$ SRA $=$ SRR12327118 - SRR12327132囚15 objects $\rrbracket$.

\section{Authors' contributions}

M.W.Z., H.Z. and Y.L. conceived and designed the study, Z.H and Y.L. coordinated the project and partly wrote the manuscript. H.Z., Y.L. and M.J.H performed animals management and bio-samples collection. H.Z., Y.L., M.J.H, N.L., Y.Y.Y., H.Y.X. X.X.L designed analyses, performed bioinformatic analyses and partly wrote the manuscript. H.Z. and X.X.L. performed validation experiments. M.W.Z. supervised and performed bioinformatic analyses. H.Z. and L.Y. wrote, revised, and revised the article. All these authors read and approved the final manuscript.

\section{Authors information}

\section{Affiliations}


Department of Rehabilitation Medicine, Peking University Third Hospital, 49 North Garden Road, Beijing 100191, China

Hong Zeng, Yao Lu, Meng-jie Huang区Nan Liu, Hua-yi Xing, Xiao-xie Liu, Yan-yan Yang, Mou-wang Zhou Department of Rehabilitation Medicine, Shanghai Ninth People's Hospital Affiliated to Shanghai Jiao Tong University School of Medicine, Huangpu 200011, China. 1

Hong Zeng

\section{Corresponding author}

Correspondence to Mou-wang Zhou.

\section{Ethics declarations}

\section{Ethics approval and consent to participate}

All experimental procedures were carried out in accordance with the Chinese Guidelines of Animal Care and Welfare, and the study was approved by the Animal Welfare Ethics Branch of the Peking University Bioethics Committee.

\section{Consent for publication}

Not applicable.

\section{Competing Interest}

The authors declare that they have no competing interests.

\section{References}

1. Fakhoury M: Spinal cord injury: overview of experimental approaches used to restore locomotor activity. Reviews in the neurosciences 2015, 26(4):397-405.

2. Bravo-Esteban E, Taylor J, Aleixandre M, Simón-Martínez C, Torricelli D, Pons JL, Avila-Martín G, Galán-Arriero I, Gómez-Soriano J: Longitudinal estimation of intramuscular Tibialis Anterior 
coherence during subacute spinal cord injury: relationship with neurophysiological, functional and clinical outcome measures. Journal of neuroengineering and rehabilitation 2017, 14(1):58.

3. Fitch MT, Doller C, Combs CK, Landreth GE, Silver J: Cellular and molecular mechanisms of glial scarring and progressive cavitation: in vivo and in vitro analysis of inflammation-induced secondary injury after CNS trauma. The Journal of neuroscience : the official journal of the Society for Neuroscience 1999, 19(19):8182-8198.

4. Kjell J, Olson L: Rat models of spinal cord injury: from pathology to potential therapies. Disease models \& mechanisms 2016, 9(10):1125-1137.

5. Shultz RB, Zhong Y: Minocycline targets multiple secondary injury mechanisms in traumatic spinal cord injury. Neural regeneration research 2017, 12(5):702-713.

6. Orr MB, Gensel JC: Spinal Cord Injury Scarring and Inflammation: Therapies Targeting Glial and Inflammatory Responses. Neurotherapeutics : the journal of the American Society for Experimental NeuroTherapeutics 2018, 15(3):541-553.

7. Yılmaz T, Kaptanoğlu E: Current and future medical therapeutic strategies for the functional repair of spinal cord injury. World journal of orthopedics 2015, 6(1):42-55.

8. Keene DL: A systematic review of the use of the ketogenic diet in childhood epilepsy. Pediatric neurology 2006, 35(1):1-5.

9. Stafstrom CE, Rho JM: The ketogenic diet as a treatment paradigm for diverse neurological disorders. Frontiers in pharmacology 2012, 3:59.

10. Winesett SP, Bessone SK, Kossoff EH: The ketogenic diet in pharmacoresistant childhood epilepsy. Expert review of neurotherapeutics 2015, 15(6):621-628.

11. Paoli A, Bianco A, Damiani E, Bosco G: Ketogenic diet in neuromuscular and neurodegenerative diseases. BioMed research international 2014, 2014:474296.

12. Shaafi S, Sharifi-Bonab M, Ghaemian N, Mokhtarkhani M, Akbari H: Early Motor-Behavioral Outcome of Ischemic Stroke with Ketogenic Diet Preconditioning: Interventional Animal Study. Journal of stroke and cerebrovascular diseases : the official journal of National Stroke Association 2019, 28(4):1032-1039.

13. McDougall A, Bayley M, Munce SE: The ketogenic diet as a treatment for traumatic brain injury: a scoping review. Brain injury 2018, 32(4):416-422.

14. Streijger F, Plunet WT, Lee JH, Liu J, Lam CK, Park S, Hilton BJ, Fransen BL, Matheson KA, Assinck P et al: Ketogenic diet improves forelimb motor function after spinal cord injury in rodents. PloS one 2013, 8(11):e78765.

15. Yarar-Fisher C, Kulkarni A, Li J, Farley P, Renfro C, Aslam H, Bosarge P, Wilson L, Barnes S: Evaluation of a ketogenic diet for improvement of neurological recovery in individuals with acute spinal cord injury: a pilot, randomized safety and feasibility trial. Spinal cord series and cases 2018, 4:88.

16. Liu Q, Wang X, Huang Z, Liu J, Ding J, Xu X, Kong G, Wu X, Yang Z, Zhu Q: Ketogenic diet delays spinal fusion and decreases bone mass in posterolateral lumbar spinal fusion: an in vivo rat model. Acta neurochirurgica 2018, 160(10):1909-1916. 
17. Lu Y, Yang YY, Zhou MW, Liu N, Xing HY, Liu XX, Li F: Ketogenic diet attenuates oxidative stress and inflammation after spinal cord injury by activating Nrf2 and suppressing the NF-KB signaling pathways. Neuroscience letters 2018, 683:13-18.

18. Wang X, Wu X, Liu Q, Kong G, Zhou J, Jiang J, Wu X, Huang Z, Su W, Zhu Q: Ketogenic Metabolism Inhibits Histone Deacetylase (HDAC) and Reduces Oxidative Stress After Spinal Cord Injury in Rats. Neuroscience 2017, 366:36-43.

19. Kong G, Huang Z, Ji W, Wang X, Liu J, Wu X, Huang Z, Li R, Zhu Q: The Ketone Metabolite $\beta$ Hydroxybutyrate Attenuates Oxidative Stress in Spinal Cord Injury by Suppression of Class I Histone Deacetylases. Journal of neurotrauma 2017, 34(18):2645-2655.

20. Yokoi A, Yoshioka Y, Yamamoto Y, Ishikawa M, Ikeda SI, Kato T, Kiyono T, Takeshita F, Kajiyama H, Kikkawa F et al: Malignant extracellular vesicles carrying MMP1 mRNA facilitate peritoneal dissemination in ovarian cancer. Nature communications 2017, 8:14470.

21. Huynh NPT, Zhang B, Guilak F: High-depth transcriptomic profiling reveals the temporal gene signature of human mesenchymal stem cells during chondrogenesis. FASEB journal : official publication of the Federation of American Societies for Experimental Biology 2019, 33(1):358-372.

22. Martin K, Jackson CF, Levy RG, Cooper PN: Ketogenic diet and other dietary treatments for epilepsy. The Cochrane database of systematic reviews 2016, 2:Cd001903.

23. Li RJ, Liu Y, Liu HQ, Li J: Ketogenic diets and protective mechanisms in epilepsy, metabolic disorders, cancer, neuronal loss, and muscle and nerve degeneration. Journal of food biochemistry 2020, 44(3):e13140.

24. Veyrat-Durebex C, Reynier P, Procaccio V, Hergesheimer R, Corcia P, Andres CR, Blasco H: How Can a Ketogenic Diet Improve Motor Function? Frontiers in molecular neuroscience 2018, 11:15.

25. Demirel A, Li J, Morrow C, Barnes S, Jansen J, Gower B, Kirksey K, Redden D, Yarar-Fisher C: Evaluation of a ketogenic diet for improvement of neurological recovery in individuals with acute spinal cord injury: study protocol for a randomized controlled trial. Trials 2020, 21(1):372.

26. Lee PR, Kossoff EH: Dietary treatments for epilepsy: management guidelines for the general practitioner. Epilepsy \& behavior: E\&B 2011, 21(2):115-121.

27. Stafstrom CE, Rho JM: The ketogenic diet as a treatment paradigm for diverse neurological disorders. Frontiers in pharmacology 2012, 3:59.

28. Broom GM, Shaw IC, Rucklidge JJ: The ketogenic diet as a potential treatment and prevention strategy for Alzheimer's disease. Nutrition (Burbank, Los Angeles County, Calif) 2019, 60:118-121.

29. Webster CC, Murphy TE, Larmuth KM, Noakes TD, Smith JA: Diet, Diabetes Status, and Personal Experiences of Individuals with Type 2 diabetes Who Self-Selected and Followed a Low Carbohydrate High Fat diet. Diabetes, metabolic syndrome and obesity : targets and therapy 2019, 12:2567-2582.

30. Burke LM: Ketogenic low-CHO, high-fat diet: the future of elite endurance sport? The Journal of physiology 2020.

31. Cerqueira NM, Oliveira EF, Gesto DS, Santos-Martins D, Moreira C, Moorthy HN, Ramos MJ, Fernandes PA: Cholesterol Biosynthesis: A Mechanistic Overview. Biochemistry 2016, 55(39):5483-5506. 
32. Straub RH, Cutolo M: Glucocorticoids and chronic inflammation. Rheumatology (Oxford, England) 2016, 55(suppl 2):ii6-ii14.

33. Ur Rahman S, Ismail M, Khurram M, Ullah I, Rabbi F, Iriti M: Bioactive Steroids and Saponins of the Genus Trillium. Molecules (Basel, Switzerland) 2017, 22(12).

34. Koper JW, Lopes-Cardozo M, Van Golde LM: Preferential utilization of ketone bodies for the synthesis of myelin cholesterol in vivo. Biochimica et biophysica acta 1981, 666(3):411-417.

35. Saher G, Brügger B, Lappe-Siefke C, Möbius W, Tozawa R, Wehr MC, Wieland F, Ishibashi S, Nave KA: High cholesterol level is essential for myelin membrane growth. Nature neuroscience 2005, 8(4): 468475.

36. Walton CM, Perry K, Hart RH, Berry SL, Bikman BT: Improvement in Glycemic and Lipid Profiles in Type 2 Diabetics with a 90-Day Ketogenic Diet. J Diabetes Res 2019, 2019: 8681959.

37. Ellenbroek JH, van Dijck L, Töns HA, Rabelink TJ, Carlotti F, Ballieux BE, de Koning EJ: Long-term ketogenic diet causes glucose intolerance and reduced $\beta$ - and $\alpha$-cell mass but no weight loss in mice. American journal of physiology Endocrinology and metabolism 2014, 306(5):E552-558.

38. Paoli A, Bianco A, Grimaldi KA, Lodi A, Bosco G: Long term successful weight loss with a combination biphasic ketogenic Mediterranean diet and Mediterranean diet maintenance protocol. Nutrients 2013, 5(12):5205-5217.

39. Stumpf SK, Berghoff SA, Trevisiol A, Spieth L, Düking T, Schneider LV, Schlaphoff L, DrehaKulaczewski S, Bley A, Burfeind D et al: Ketogenic diet ameliorates axonal defects and promotes myelination in Pelizaeus-Merzbacher disease. Acta neuropathologica 2019, 138(1):147-161.

40. Zeng H, Liu N, Yang YY, Xing HY, Liu XX, Li F, La GY, Huang MJ, Zhou MW: Lentivirus-mediated downregulation of a-synuclein reduces neuroinflammation and promotes functional recovery in rats with spinal cord injury. Journal of neuroinflammation 2019, 16(1):283.

41. Milich LM, Ryan CB, Lee JK: The origin, fate, and contribution of macrophages to spinal cord injury pathology. Acta neuropathologica 2019, 137(5):785-797.

42. Li Y, Gu R, Zhu Q, Liu J: Changes of Spinal Edema and Expression of Aquaporin 4 in Methylprednisolone-treated Rats with Spinal Cord Injury. Annals of clinical and laboratory science 2018, 48(4):453-459.

43. Lee JY, Choi HY, Ju BG, Yune TY: Estrogen alleviates neuropathic pain induced after spinal cord injury by inhibiting microglia and astrocyte activation. Biochimica et biophysica acta Molecular basis of disease 2018, 1864(7): 2472-2480.

44. Zendedel A, Mönnink F, Hassanzadeh G, Zaminy A, Ansar MM, Habib P, Slowik A, Kipp M, Beyer C: Estrogen Attenuates Local Inflammasome Expression and Activation after Spinal Cord Injury. Molecular neurobiology 2018, 55(2): 1364-1375.

45. Chen XB, Wang ZL, Yang QY, Zhao FY, Qin XL, Tang XE, Du JL, Chen ZH, Zhang K, Huang FJ: Diosgenin Glucoside Protects against Spinal Cord Injury by Regulating Autophagy and Alleviating Apoptosis. International journal of molecular sciences 2018, 19(8). 
46. Zhang Z, Chen J, Chen F, Yu D, Li R, Lv C, Wang H, Li H, Li J, Cai Y: Tauroursodeoxycholic acid alleviates secondary injury in the spinal cord via up-regulation of CIBZ gene. Cell stress \& chaperones 2018, 23(4):551-560.

47. Trapnell C, Pachter L, Salzberg SL: TopHat: discovering splice junctions with RNA-Seq. Bioinformatics (Oxford, England) 2009, 25(9):1105-1111.

48. Li B, Dewey CN: RSEM: accurate transcript quantification from RNA-Seq data with or without a reference genome. BMC bioinformatics 2011, 12:323.

49. Love MI, Huber W, Anders S: Moderated estimation of fold change and dispersion for RNA-seq data with DESeq2. Genome biology 2014, 15(12):550.

50. Buchfink B, Xie C, Huson DH: Fast and sensitive protein alignment using DIAMOND. Nature methods 2015, 12(1):59-60.

51. Powell S, Forslund K, Szklarczyk D, Trachana K, Roth A, Huerta-Cepas J, Gabaldón T, Rattei T, Creevey C, Kuhn M et al: eggNOG v4.0: nested orthology inference across 3686 organisms. Nucleic acids research 2014, 42(Database issue):D231-239.

52. Finn RD, Clements J, Eddy SR: HMMER web server: interactive sequence similarity searching. Nucleic acids research 2011, 39(Web Server issue):W29-37.

53. Xie C, Mao X, Huang J, Ding Y, Wu J, Dong S, Kong L, Gao G, Li CY, Wei L: KOBAS 2.0: a web server for annotation and identification of enriched pathways and diseases. Nucleic acids research 2011, 39(Web Server issue):W316-322.

54. Teng L, Fan X, Xu D, Zhang X, Mock T, Ye N: Identification of Genes under Positive Selection Reveals Differences in Evolutionary Adaptation between Brown-Algal Species. Frontiers in plant science 2017, 8:1429.

55. Szklarczyk D, Morris JH, Cook H, Kuhn M, Wyder S, Simonovic M, Santos A, Doncheva NT, Roth A, Bork P et al: The STRING database in 2017: quality-controlled protein-protein association networks, made broadly accessible. Nucleic acids research 2017, 45(D1):D362-d368.

\section{Figures}




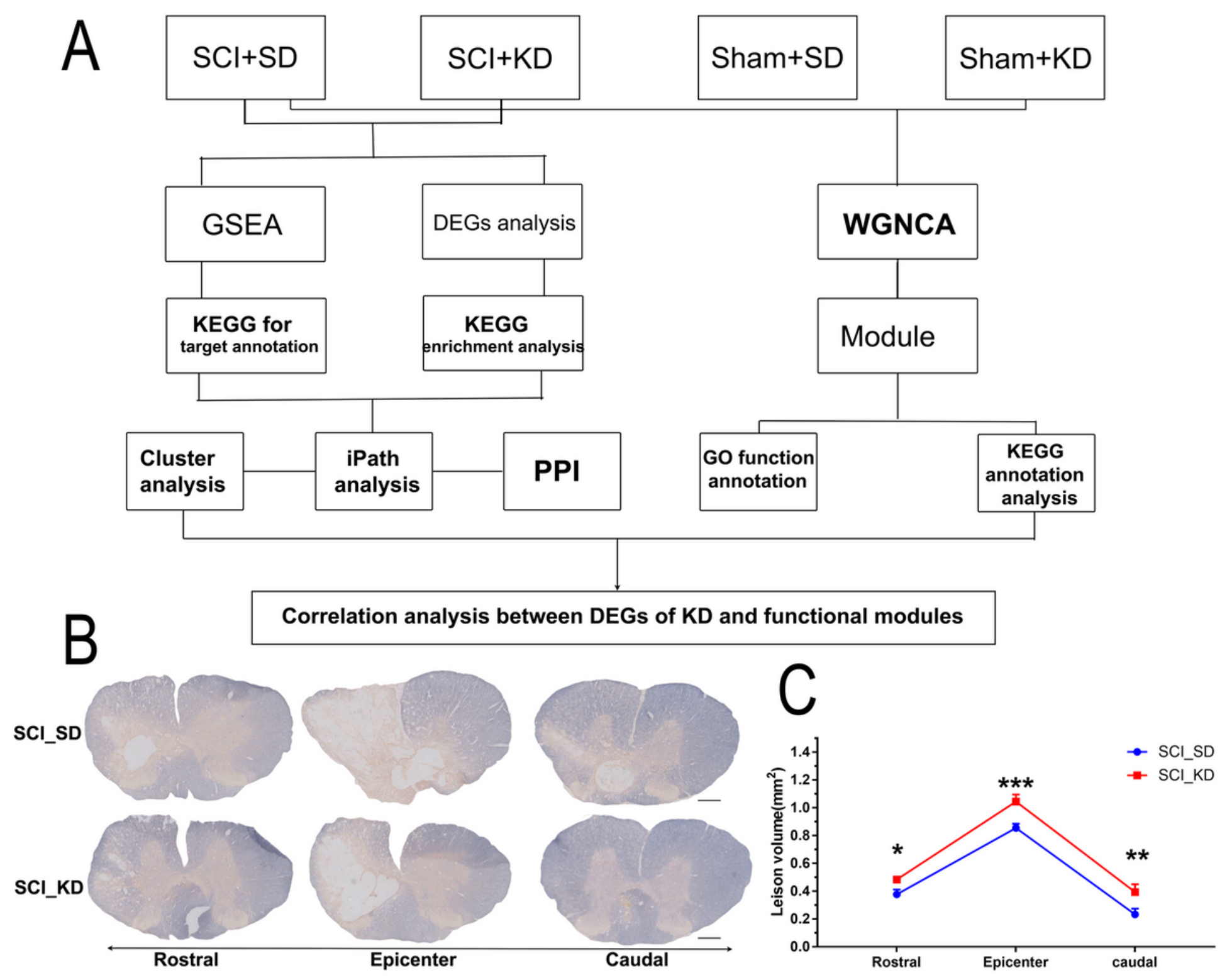

Figure 1

Experimental protocol and animal myelin area. (A) Detailed experimental protocol. (B) Representative images showing EC staining after SCl. Scale bar $=1 \mathrm{~mm}$. (C) Quantitative analysis of the results in panel (A). All data are presented as the mean $\pm S D, n=3$. Rostral of the injury site, $p=0.0255$; Epicenter, $p=0.0004$; Caudal, $p=0.0015$. 


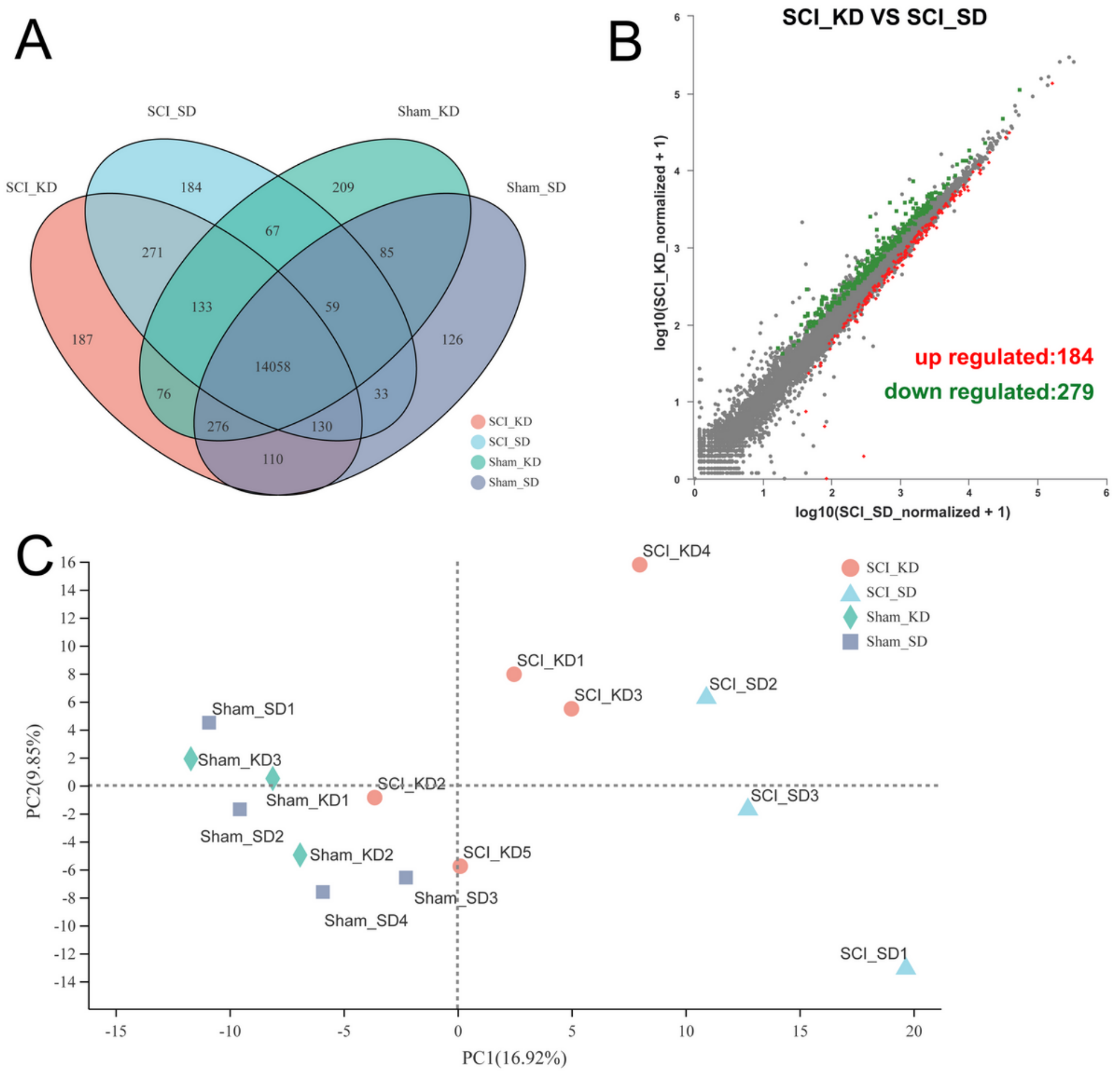

\section{Figure 2}

Changes in mRNA expression profiles between groups. (A) The mRNA expression of the four groups in the entire transcriptome. (B) Volcano plots showing the up- and downregulated mRNA transcripts in the SCl_KD vs SCl_SD group. (C) Principal component analysis (PCA) score plot with the two principal components (PC1=16.92\%; PC2 =9.85\%). 
A Top 20 of KEGG enrichment (up regulated DEGs)

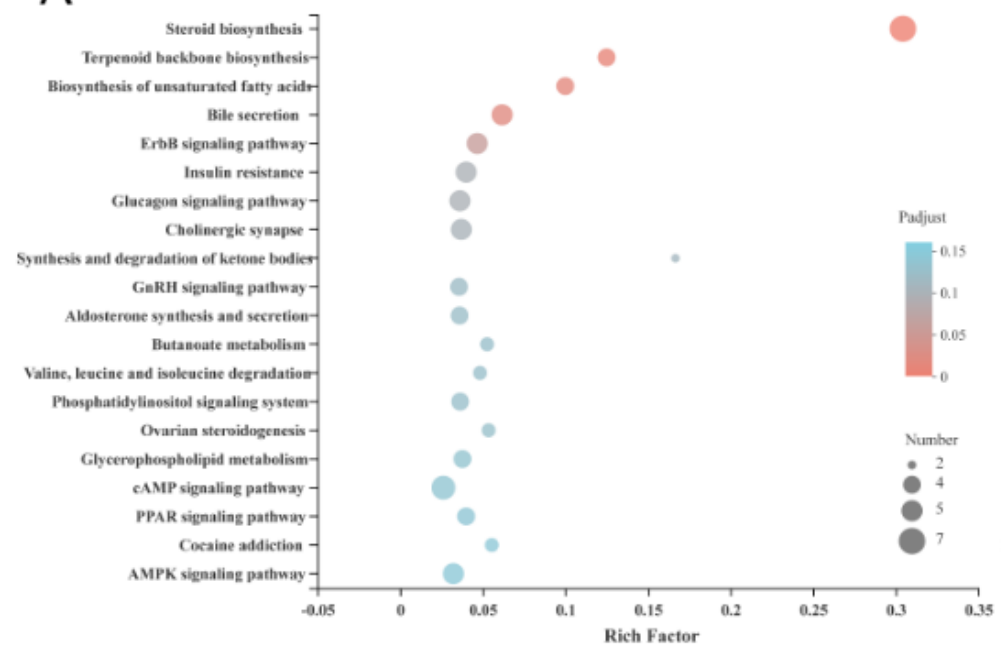

B Top 20 of KEGG enrichment (down regulated DEGs)

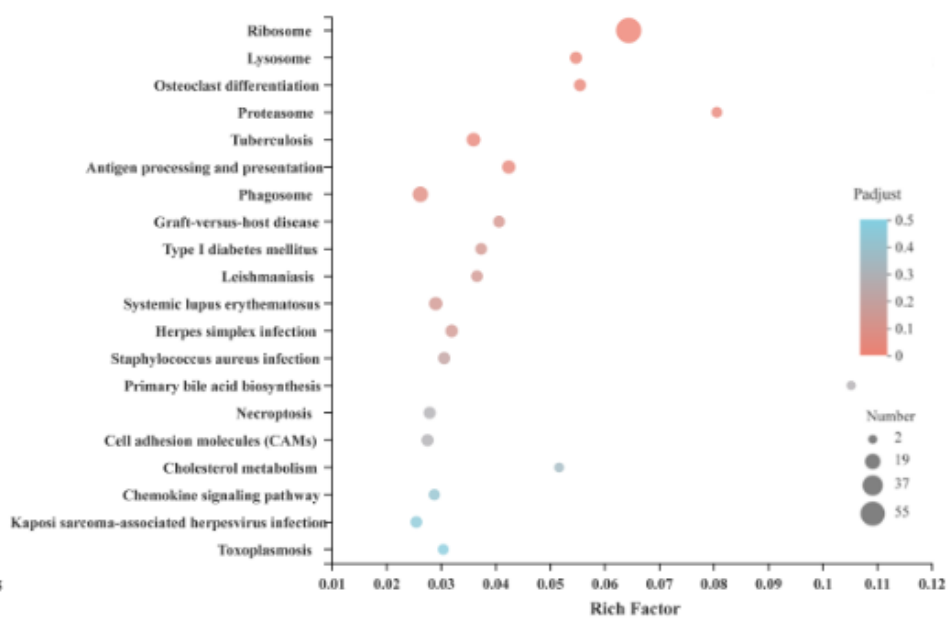

C

Steroid Biosynthesis
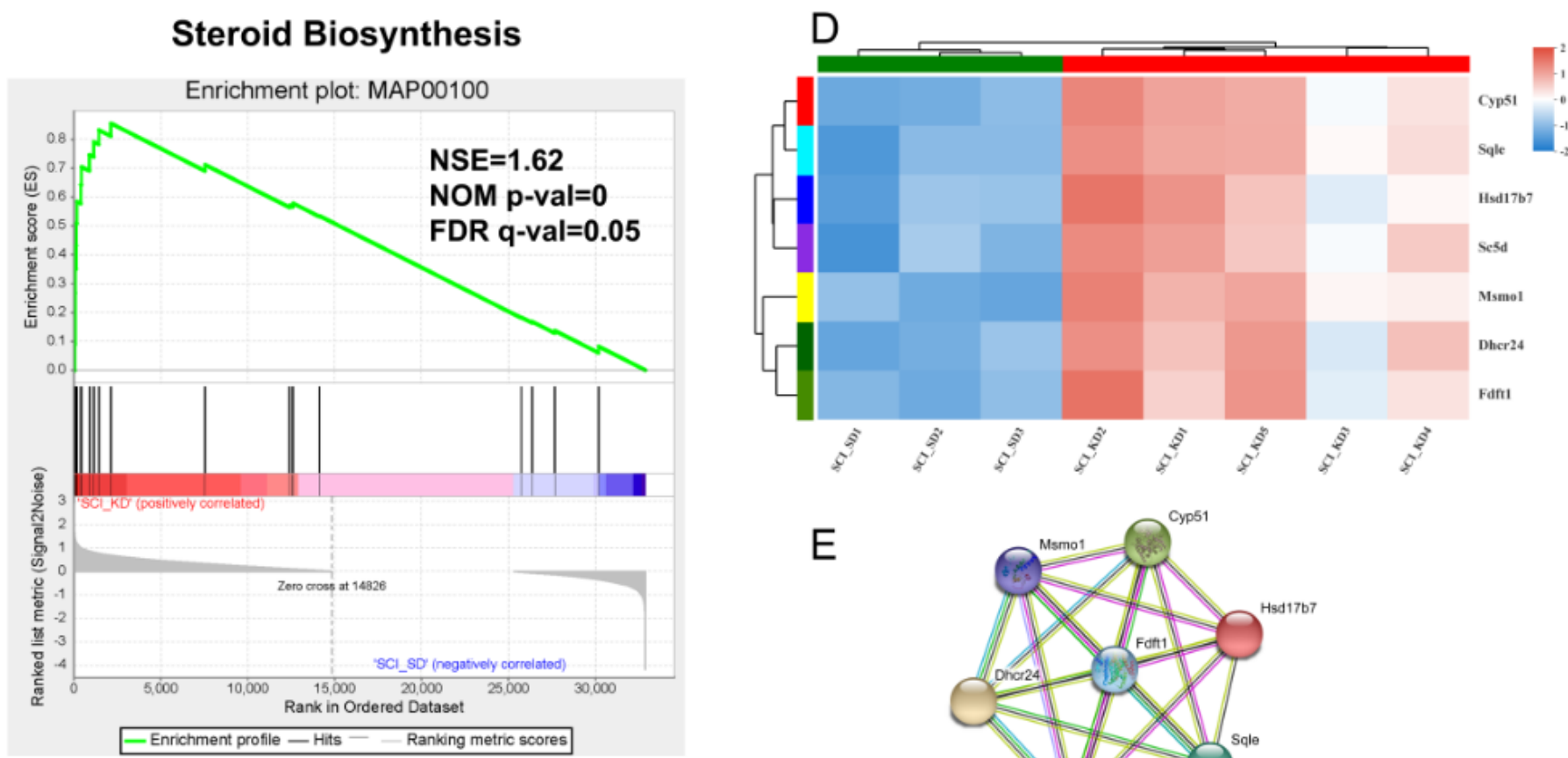

$\mathrm{E}$

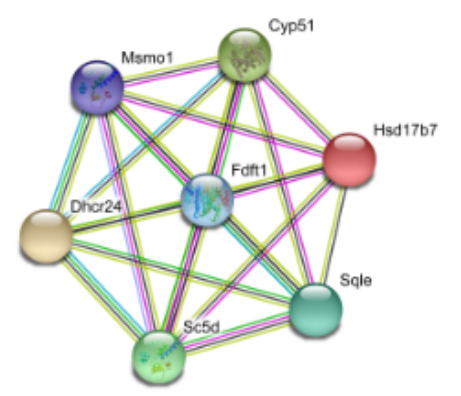

Figure 3

Enrichment Analysis of DEGs in the SCI_KD vs SCI_SD group. (A) KEGG enrichment bubble diagram of the upregulated DEGs from the SCI_KD and SCI_SD groups. (B) KEGG enrichment bubble diagram of the downregulated DEGs from the SCI_KD and SCI_SD groups. (C) The GESA analysis diagram of steroid biosynthesis between SCI_KD and SCI_SD. (D) Cluster analysis of KEGG-enriched DEGs. (E) Proteinprotein interaction of DEGs between the SCI_KD and SCI_SD groups. 
A

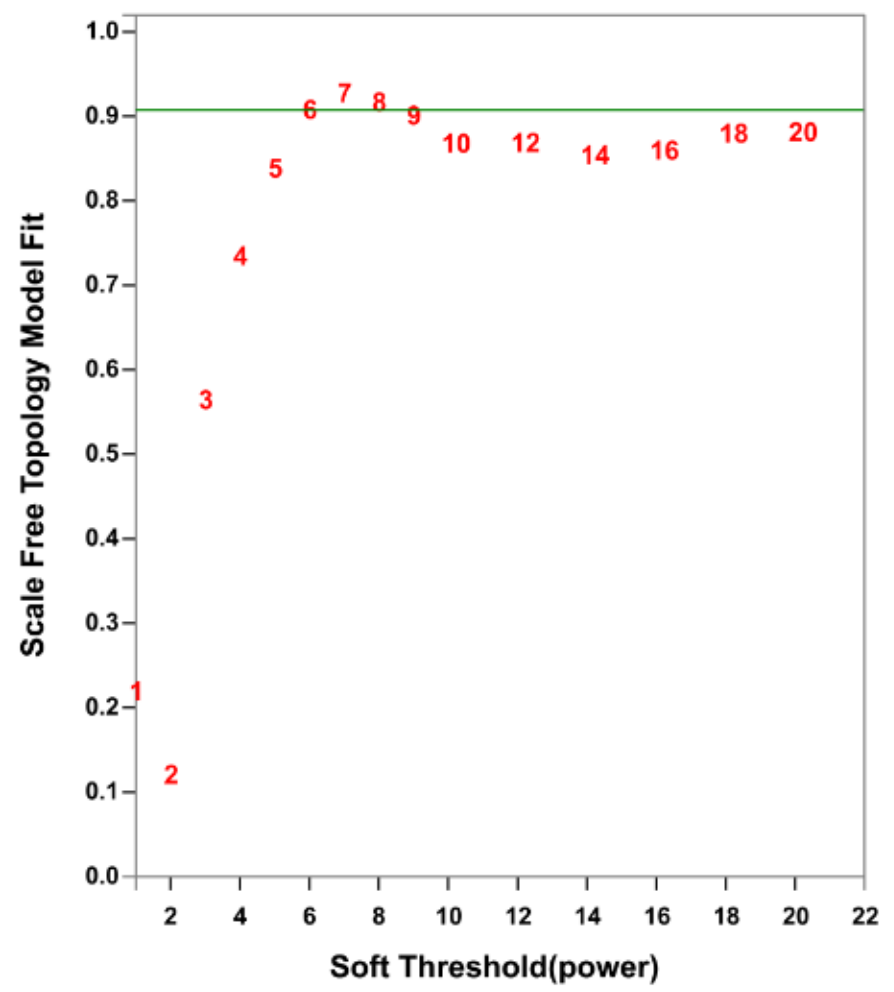

B

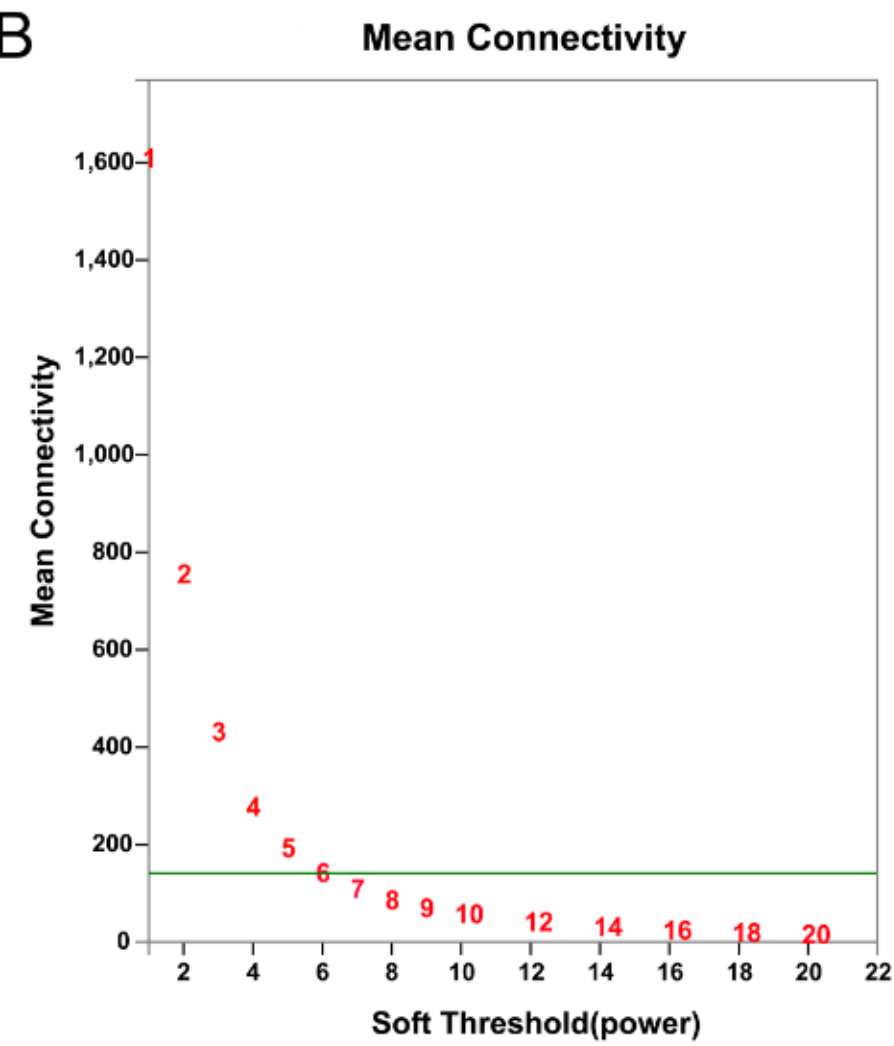

D Eigengene adiacency heatmap

Correlation between module and trait

C

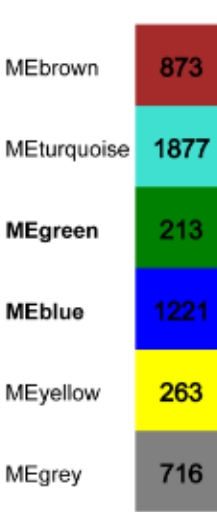

716

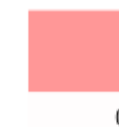

(0.131)

$(0.974)$

$-0.48$

$(0.0702)$

0.347

(0.205)

0.298

(0.281)

$-0.232$

(0.405)

SCI_KD

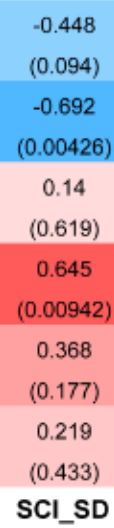

SCI_SD

\section{$-0.242$}

$(0.385)$

0.509

$(0.0526)$

0.806

$(0.000285)$

$-0.416$

$(0.123)$

$-0.208$

$(0.457)$

0.608

(0.0162)

Sham_KD

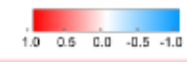

0.189

(0.5)

0.156

(0.579)

$-0.345$

(0.208)

$-0.578$

$(0.024)$

$-0.463$

(0.0822)

$-0.5$

(0.0577)

Sham_SD

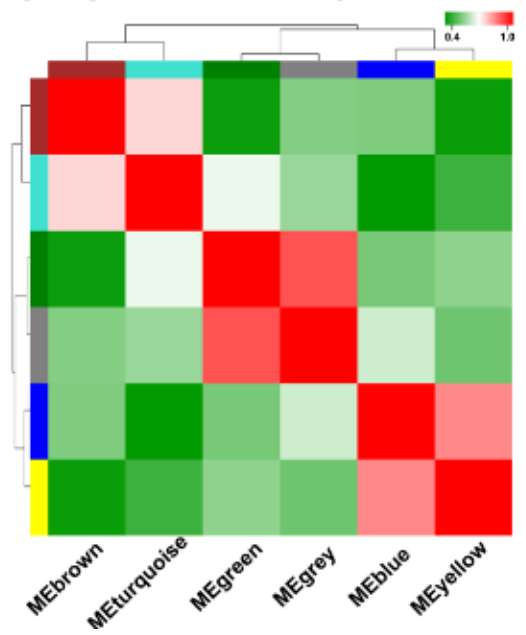

Figure 4

Data pre-processing and module identifying for WGCNA analysis. (A-B) Analysis of the network topology with different soft threshold powers. The left panel shows the influence of soft threshold power on the scale-free topology fitting index. The right panel shows the influence of soft threshold power on the average connectivity. (C) Analysis of the correlation between the module and the diet phenotype. Red means the module has a higher correlation with the phenotype and blue means the module has a lower correlation with the phenotype. (D) WGCNA module correlation; the colour indicates the correlation between modules. 
A

or biogenesis

localization

response to stimulus

developmental process

metabolic process

biological regulation cellular process

extracellular region

extracellular region part

protein-containing complex

membrane part

organelle part

membrane

organelle

cell part

transcription regulator activity

transporter activity

catalytic activity

binding

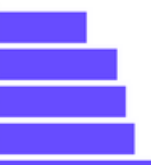

molecular_function

cellular_component

biological_process

B

multi-organism process multicellular organismal process

localization

cellular component organization or biogenesis

immune system process

developmental process

response to stimulus

metabolic process

biological regulation

cellular process

protein-containing complex

extracellular region part

organelle part

membrane part

membrane

organelle

cell part

molecular function regulator

catalytic activity

binding

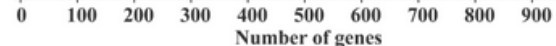

Figure 5

GO function annotation analysis of WGCNA module genes. (A) The GO function annotation analysis histogram of the MEgreen module. (B) The GO function annotation analysis histogram of the MEblue module, includes biological processes, cell components, and molecular functions. 


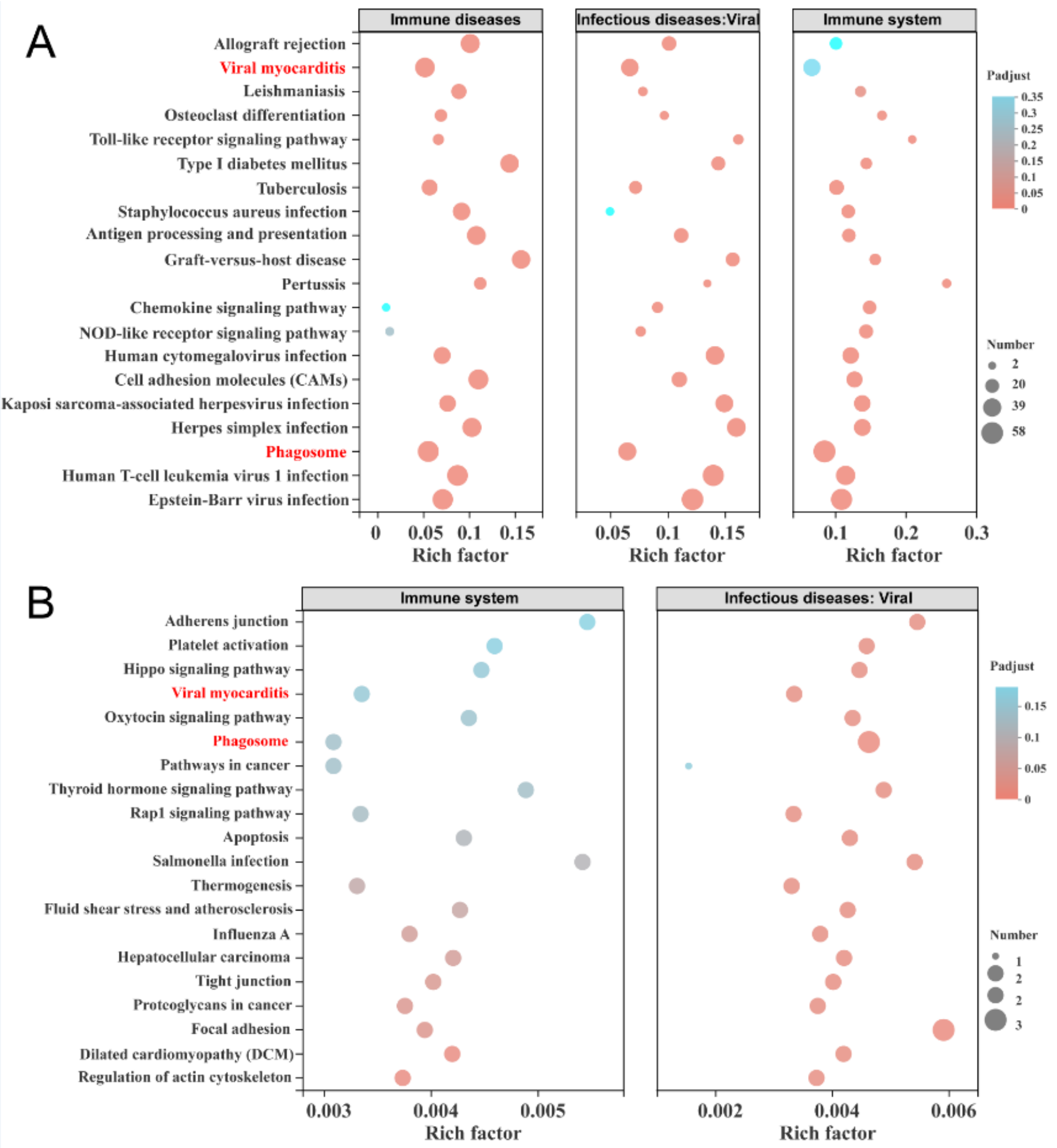

\section{Figure 6}

Multi-gene set KEGG enrichment analysis and modular gene visualization. (A) The bubble diagram of the intersection of the KEGG pathway in the MEblue module, including the Immune system and Infectious diseases: Viral and Immune diseases gene sets. (B) The bubble diagram of the intersection of the KEGG pathway in the MEgreen module, including the Immune system and Infectious diseases: Viral gene sets. 


\section{Supplementary Files}

This is a list of supplementary files associated with this preprint. Click to download.

- Additionalfile10.tif

- Additionalfile9.xls

- Additionalfile8.tif

- Additionalfile7.pdf

- Additionalfile6.tif

- Additionalfile5.xIs

- Additionalfile4.xls

- Additionalfile3.xls

- Additionalfile2.xls

- Additionalfile1.tif 